\title{
Caste-specific and sex-specific expression of chemoreceptor genes in a termite
}

\section{$\operatorname{AUTHOR}(\mathrm{S}):$}

Mitaka, Yuki; Kobayashi, Kazuya; Mikheyev, Alexander; Tin, Mandy M. Y.; Watanabe, Yutaka; Matsuura, Kenji

\section{CITATION:}

Mitaka, Yuki ... [et al]. Caste-specific and sex-specific expression of chemoreceptor genes in a termite. PLOS ONE 2016, 11(1): e0146125.

\section{ISSUE DATE:}

2016-01-13

URL:

http://hdl.handle.net/2433/212047

\section{RIGHT:}

(C) 2016 Mitaka et al. This is an open access article distributed under the terms of the Creative Commons Attribution License, which permits unrestricted use, distribution, and reproduction in any medium, provided the original author and source are credited. 


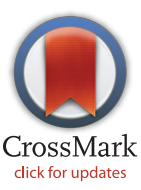

\section{OPEN ACCESS}

Citation: Mitaka Y, Kobayashi K, Mikheyev A, Tin MMY, Watanabe Y, Matsuura K (2016) Caste-Specific and Sex-Specific Expression of Chemoreceptor Genes in a Termite. PLoS ONE 11(1): e0146125. doi:10.1371/journal.pone. 0146125

Editor: Joseph Clifton Dickens, United States Department of Agriculture, Beltsville Agricultural Research Center, UNITED STATES

Received: September 20, 2015

Accepted: December 14, 2015

Published: January 13, 2016

Copyright: @ 2016 Mitaka et al. This is an open access article distributed under the terms of the Creative Commons Attribution License, which permits unrestricted use, distribution, and reproduction in any medium, provided the original author and source are credited.

Data Availability Statement: RNA sequence data was deposited in the DNA Data Bank of Japan (DDBJ) under the BioProject "Royal epigenetics in the termite Reticulitermes speratus" (PRJDB3531), which contains links and access to insect sampling data through the BioSample SAMD00026264SAMD00026323 and the Sequence Read Archive DRR030795-DRR030854. The predicted mRNA sequences of chemoreceptor genes are available at DDBJ (FX982902-FX982954). These data will only be available after acceptance
RESEARCH ARTICLE

\section{Caste-Specific and Sex-Specific Expression of Chemoreceptor Genes in a Termite}

\author{
Yuki Mitaka $^{10}$, Kazuya Kobayashi $^{10}$, Alexander Mikheyev $^{2}$, Mandy M. Y. Tin ${ }^{2}$, \\ Yutaka Watanabe $^{2}$, Kenji Matsuura ${ }^{*}$ * \\ 1 Laboratory of Insect Ecology, Division of Applied Biosciences, Graduate School of Agriculture, Kyoto \\ University, Kyoto, 606-8502, Japan, 2 Ecology and Evolution Unit, Okinawa Institute of Science and \\ Technology Graduate University, Okinawa, 904-0495, Japan \\ ๑ These authors contributed equally to this work. \\ * kenjijpn@kais.kyoto-u.ac.jp
}

\section{Abstract}

The sophisticated colony organization of eusocial insects is primarily maintained through the utilization of pheromones. The regulation of these complex social interactions requires intricate chemoreception systems. The recent publication of the genome of Zootermopsis nevadensis opened a new avenue to study molecular basis of termite caste systems. Although there has been a growing interest in the termite chemoreception system that regulates their sophisticated caste system, the relationship between division of labor and expression of chemoreceptor genes remains to be explored. Using high-throughput mRNA sequencing (RNA-seq), we found several chemoreceptors that are differentially expressed among castes and between sexes in a subterranean termite Reticulitermes speratus. In total, 53 chemoreception-related genes were annotated, including 22 odorant receptors, 7 gustatory receptors, 12 ionotropic receptors, 9 odorant-binding proteins, and 3 chemosensory proteins. Most of the chemoreception-related genes had caste-related and sex-related expression patterns; in particular, some chemoreception genes showed king-biased or queen-biased expression patterns. Moreover, more than half of the genes showed significant age-dependent differences in their expression in female and/or male reproductives. These results reveal a strong relationship between the evolution of the division of labor and the regulation of chemoreceptor gene expression, thereby demonstrating the chemical communication and underlining chemoreception mechanism in social insects.

\section{Introduction}

Animals perceive chemical signals in their environment using chemical senses (olfaction and gustation), mainly to locate potential food sources, detect predators, and to receive chemical cues in social interactions [1]. A chemical signal used to communicate among members of the same species is called a pheromone [2]. The sophisticated colony organization of eusocial insects is primarily maintained through the utilization of pheromones, which are also likely involved in all social activities including foraging, sexual behavior, defense, nestmate 
Funding: This work was supported by Japan Society for the Promotion of Science (https://www.jsps.go.jp/ english/index.html, No. 25221206 to KM).

Competing Interests: The authors have declared that no competing interests exist. recognition, and caste regulation [3]. The extraordinary diversity of pheromones is the evolutionary consequence of the powerful and flexible way that the chemoreception system is organized [1].

Division of labor is one of the most prominent features of colony behavior in social insects [4]. Individuals in these societies interact via a complex web of chemical signals. Studies of division of labor have typically been concerned with the integration of individual worker behavior into colony-level task organization, and with the question of how it is regulated by chemical communication [5]. The regulation of complex social interactions requires intricate systems of chemical communication [6], and the evolution of division of labor should be strongly related to the evolution of chemoreception systems. It is reasonably predicted that individuals engaging in different tasks can differ in their sensitivity to various chemicals, and thus may have different sets of chemoreceptors. However, the relationship between caste differentiation and the differential expression of chemoreceptor genes is not well understood.

Eusociality in Isoptera (termites) converges along many lines of colony organization and social behavior in eusocial Hymenoptera (ants, bees, and wasps). Unlike social Hymenoptera, however, termites of both sexes are diploid, and their societies consist of both sexes of helpers (workers and soldiers) and reproductives (kings and queens). In termites, pheromones produced by reining queens inhibit the differentiation of new reproductive individuals [7-9]. Recently, a termite queen pheromone consisting of a volatile blend of n-butyl-n-butyrate $(\mathrm{nBnB})$ and 2-methyl-1-butanol (2M1B) was identified in the subterranean termite Reticulitermes speratus [10]. Moreover, the breeding system of $R$. speratus is characterized by asexual queen succession (AQS) (i.e., queens produce their neotenic replacements asexually, but use normal sexual reproduction to produce other colony members) [11,12] (Fig 1). The perception of the inhibitory queen pheromone may be involved in the underling mechanism through which asexually produced daughters have a developmental priority to be neotenic queens [13]. Therefore, R. speratus provides an ideal system to understand how chemical communications regulate the sophisticated caste system in termites. There has been a growing interest in the chemoreception system of termites. Recently, the genome of a dampwood termite Zootermopsis nevadensis was sequenced, which first provided the list of chemoreceptor genes in termites [14]. However, there is no information on the differential expression of chemoreceptor genes among castes and between sexes in termites.

Here, we assembled and analyzed transcriptomes from next-generation sequencing of all castes of $R$. speratus including the mature primary king, secondary queen, and both sexes of alates, founders (young primary kings and queens), soldiers, and workers to identify chemoreception-related genes (Fig 2) and compare their expression among castes and sexes. From the obtained mRNA library, the candidate genes of the odorant receptor (OR), gustatory receptor (GR), ionotropic receptor (IR), odorant-binding protein (OBP), and chemosensory protein (CSP) were annotated. Subsequently, the expression of each chemoreception-related gene was compared among castes and between sexes.

\section{Results}

\section{Transcriptome sequencing and de novo transcriptome assembly}

Illumina sequencing of all castes and sexes in R. speratus yielded a total of $729 \mathrm{M}$ read pairs with an average length of $93 \mathrm{bp}$ for each short read, and the percentage of GC content was $41 \%$. The transcriptome was assembled de novo with Trinity $[19,20]$ and yielded a total of 856 Mbp of mRNA sequences, 1,144,272 contigs with a minimum length of $201 \mathrm{bp}$, a median length of $360 \mathrm{bp}$, a maximum length of $35,267 \mathrm{bp}$, a N50 value of 1,296 bp, and an average length of $748 \mathrm{bp}$ (S1 Fig and Table 1). 

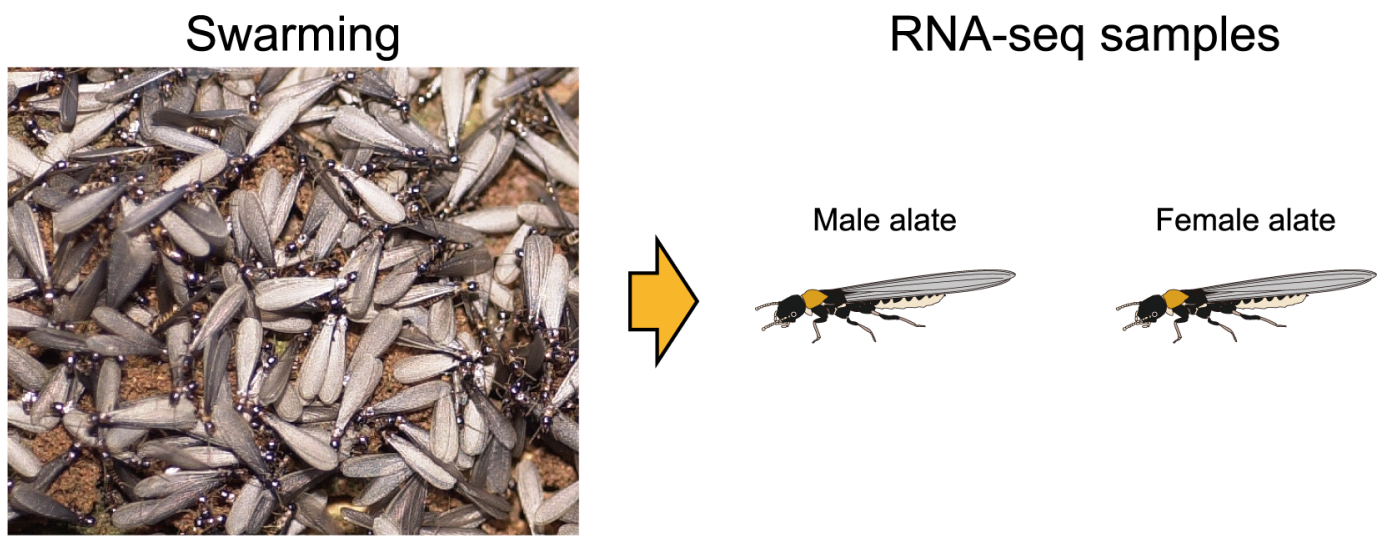

\section{Incipient colony}
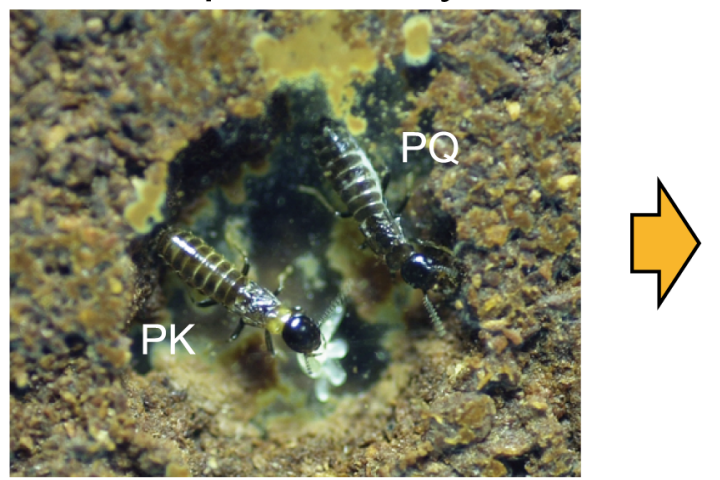

Young primary king

Young primary queen
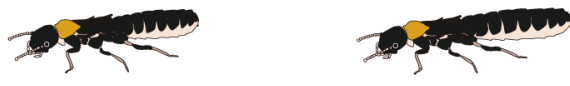

\section{Mature colony}
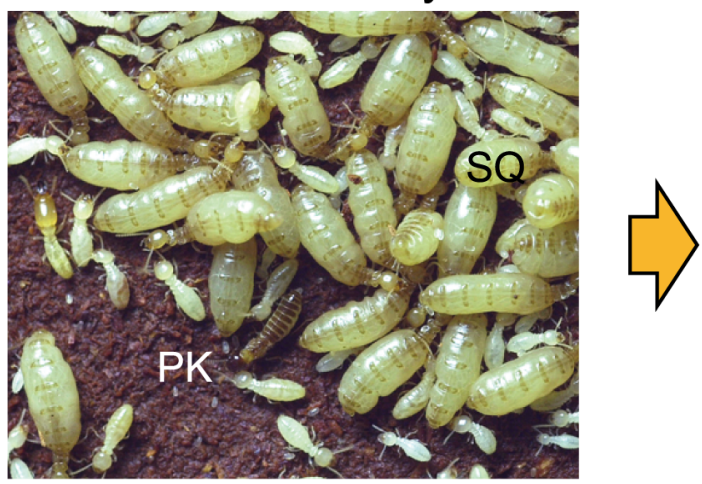

Mature primary king

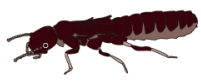

Male soldier

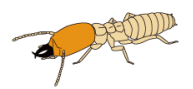

Male worker

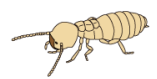

Mature secondary queen

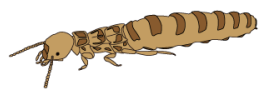

Female soldier

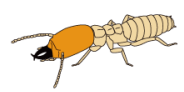

Female worker

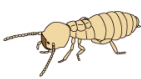

Fig 1. Life history of $R$. speratus and RNA-seq samples. Each colony of $R$. speratus produces innumerable alates in spring. After swarming, a pair of male and female alates establishes a new colony, and starts to produce offspring sexually as a primary king (PK) and a primary queen (PQ). As the PQ senesces, secondary queens $(\mathrm{SQ}$ ) produced asexually by the $\mathrm{PQ}$ differentiate within the colony and supplement egg production, eventually replacing the $\mathrm{PQ}$. For $\mathrm{RNA}$ sequencing (RNA-seq) analysis, we obtained alates from three swarming colonies, and the young PK and PQ were obtained from three incipient colonies artificially established by the same colonies. Mature PK, mature $\mathrm{SQ}$, and both sexes of soldiers and workers were obtained from four large field colonies.

doi:10.1371/journal.pone.0146125.g001

Using transcriptsToOrfs, 156,276 ORFs were detected with a total length of 76,684,150 amino acids (aa), an average length of 490.70 aa, a median length of 381 aa, a maximum length of 11,369 aa, a minimum length of 100 aa, and a N50 length of 584 aa (Table 1). Based on the subsequent homology search of the known amino acid sequence data from Zootermopsis nevadensis [14], Acyrthosiphon pisum [21], Apis mellifera [22], Tribolium castaneum [23], Bombyx 
A

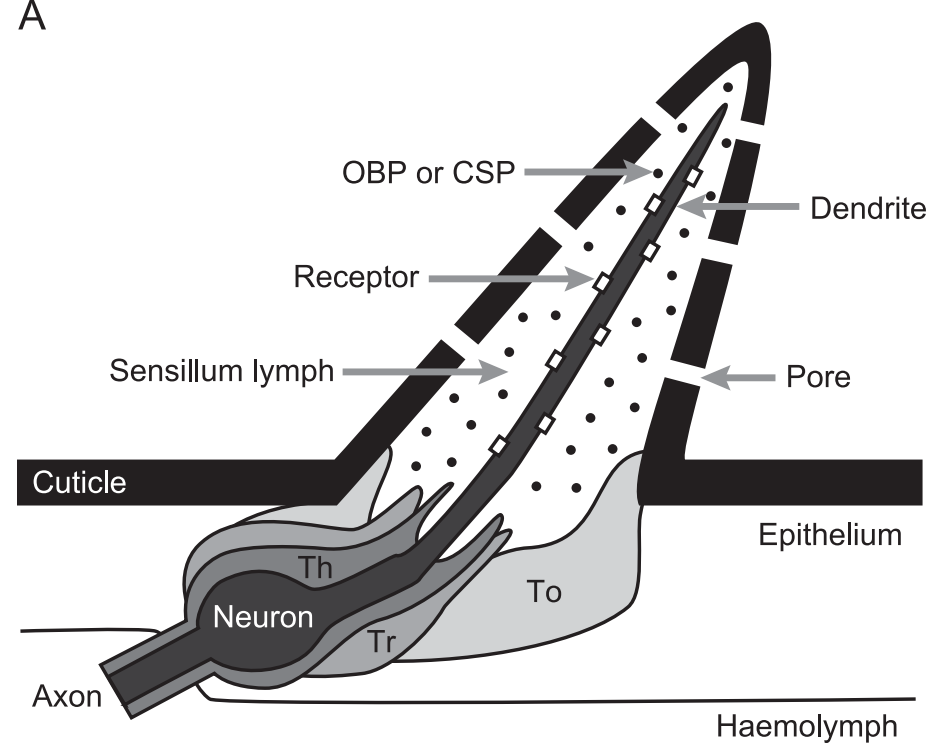

B

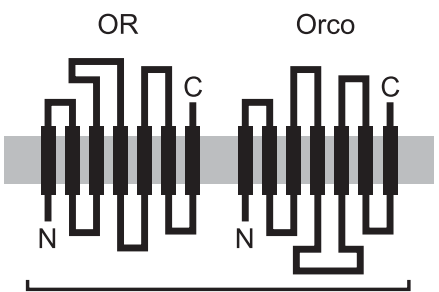

OR / Orco complex

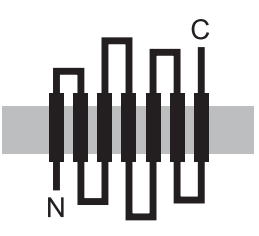

GR

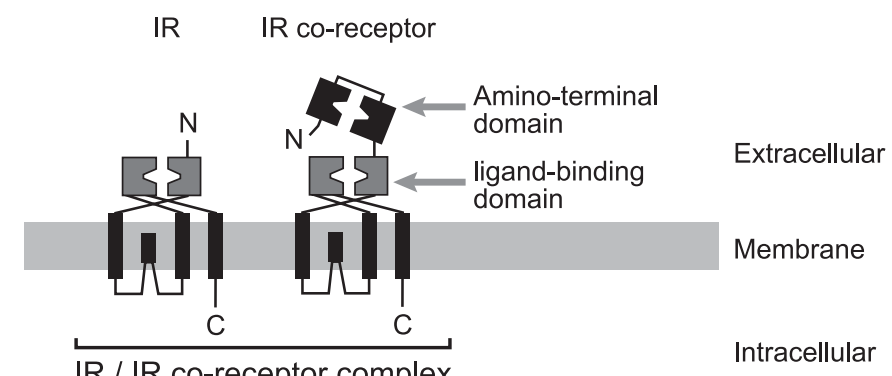

Fig 2. Details of chemoreception. (A) General structure of an insect sensillum (modified from [15]). In insects, odorants are detected by the dendrites of neuron that are housed within chemosensilla mainly on antennae and maxillary palps, and contact chemicals are detected mainly on labial palps and tarsi. The neurons are surrounded by accessory cells (To: tormogen, Th: thecogen, and Tr: trichogen cells). The dendrites of neurons are bathed in aqueous sensillar lymph that protects them from dehydration. After entering the sensillum through cuticular pores, the chemicals are transported by odorant-binding proteins (OBPs) or chemosensory proteins (CSPs) to reach the chemoreceptor on the membrane of dendrite as crossing the lymph. Each chemical binds to a corresponding chemoreceptor, thereby a chemical signal in the environment is converted into an electrical signal that can be interpreted by the insect nervous system. (B) Schematic diagrams of odorant receptor (OR), gustatory receptor (GR), and ionotropic receptor (IR) (modified from [16-18]). Insect ORs and GRs are seven-transmembrane domain proteins with a reversed membrane topology (intracellular $\mathrm{N}$-terminus) compared to vertebrate ORs. The OR determining ligand specificity forms heteromers with a highly conserved odorant receptor co-receptor (ORCO) in insects. IRs have recently been discovered as another class of receptors involved in chemoreception in $D$. melanogaster. IR forms heteromers composed of the compound-specific receptor and the coreceptor.

doi:10.1371/journal.pone.0146125.g002

mori [24], and Drosophila melanogaster [25], 10,238 protein-coding transcripts were inferred in R. speratus.

\section{Odorant receptor}

We found 22 putative ORs through a blast search with the amino acid sequences of ORs in various insect species in the query (S2 Table); all were classified as being in the superfamily of 7-transmembrane receptors (BLASTP, NCBI). RsORCO showed higher sequence similarity to the ORCO [15] of Z. nevadensis (91\%), M. caryae (64\%), A. mellifera (60\%), B. mori (59\%),

Table 1. Summary statistics of assembly.

Assembly assessment parameters

Total length

Number of gene products

Average length

Median length

Maximum length

Minimum length

N50 length

GC content

doi:10.1371/journal.pone.0146125.t001

\section{Transcriptional products}

Translation products

$856,112,139 \mathrm{bp}$
$1,144,272$
$748 \mathrm{bp}$
$360 \mathrm{bp}$
$35,267 \mathrm{bp}$
$201 \mathrm{bp}$
$1,296 \mathrm{bp}$
$41 \%$

$76,684,150$ aa

156,276

491 aa

381 aa

11,369 aa

100 aa

584 aa 
T. castaneum (64\%), Dendroctonus ponderosae (64\%), D. melanogaster (72\%), Anopheles gambiae (73\%), Aedes aegypti (72\%), and Locusta migratoria (63\%). The phylogeny inferred from the ORCO amino acid sequences was consistent with the previously known topology of the insect order (S3 Fig). The ligands of other receptors remain unknown.

The expression level of RsOr3 was zero in both sexes of alates, secondary queens, and male workers and soldiers (caste: false discovery rate $(\mathrm{FDR})<0.001$; Figs 3 and 4 and S3 Table). RsOr10 was commonly expressed in all of the castes except the male alates and secondary queens (caste: FDR $<0.001$, caste $\times$ sex: FDR $<0.05$; S3 Table). RsORCO was more highly expressed in young primary kings and queens, soldiers and workers than other castes (caste: FDR $<0.001$; Fig 3 and S2 Fig and S3 Table). Some ORs were more highly expressed in reproductive castes than in other castes (Figs 3 and 4 and S2 Fig). RsOr 5 was expressed more in young primary kings and queens (caste: FDR $<0.001$; S3 Table), whereas RsOr8 was expressed more in mature primary kings and secondary queens (caste: FDR $<0.001$, sex: FDR $<0.05$; S3 Table). Four ORs (RsOr2, RsOr7, RsOr18, and RsOr22) were expressed more in secondary queens (caste: FDR $<0.001$; S3 Table), whereas RsOr9 and RsOr16 were commonly expressed but showed differential expression among castes (caste: FDR $<0.001$, S3 Table).

\section{Gustatory receptor}

Seven GRs were predicted through a blast search with the GR sequences of D. melanogaster, $Z$. nevadensis, and T. castaneum (S2 Table), and were classified as being part of the 7-transmembrane superfamily (BLASTP, NCBI). RsGr2 was only expressed in reproductive castes, especially in young primary queens and secondary queens (caste: FDR $<0.001$; Fig 4 and S3 Table). RsGr5 and RsGr7 were expressed more in female reproductive castes (RsGr5: caste: FDR $<0.001$, sex: FDR $<0.01$, caste $\times$ sex: FDR $<0.001$, RsGr7: caste: FDR $<0.001$, sex: FDR $<0.001$, caste $\times$ sex FDR $<0.001$; Fig 4 and S3 Table). Three GRs (RsGr3, RsGr4, and RsGr6) were commonly expressed in all of the castes (Fig 3 and S4 Fig), but only RsGr3 showed no differential expression among castes (caste: n.s., sex: n.s., caste $\times$ sex: n.s.; S3 Table). RsGr1 was only expressed in male alates, young primary queens, mature primary kings, and female soldiers (caste $\times$ sex: FDR $<0.05$; S3 Table).

\section{Ionotropic receptor}

We found 12 putative IRs based on their sequence similarity to those of D. melanogaster (S2 Table); all were classified as being part of the periplasmic binding protein superfamily (BLASTP, NCBI). RsIR1 showed secondary-queen-biased expression (caste: FDR $<0.001$; Fig 4 and S3 Table), and RsIR11 showed higher expression in secondary queens and mature primary kings (caste: FDR $<0.001$, caste $\times$ sex: FDR $<0.001$; Fig 4 and S3 Table). Seven IRs (RsIR3, RsIR5, RsIR6, RsIR7, RsIR8, RsIR9, and RsIR10) had differential caste expression (caste: FDR < 0.001; S5 Fig and S3 Table), and in particular, RsIR10 also indicated a caste-sex interaction (caste $\times$ sex: FDR $<0.001 ;$ S3 Table). RsIR2 and RsIR4 were equally expressed in all of the castes (caste: n.s.; S5 Fig and S3 Table).

\section{Odorant-binding protein}

Nine OBPs were predicted, which resembled the sequences of OBPs of Periplaneta americana, D. melanogaster, A. gambiae, T. castaneum, and Rhodnius prolixus (Fig 5, S2 Table). Two OBPs (RsOBP8, and RsOBP9) were expressed more in soldiers (caste: FDR < 0.001; S6 Fig, S3 Table). Six OBPs (RsOBP1, RsOBP2, RsOBP3, RsOBP4 RsOBP6 and RsOBP7) were expressed in all of the castes except alates (caste: FDR $<0.001$; S6 Fig and S3 Table), whereas the 


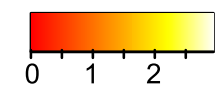

Relative expression level

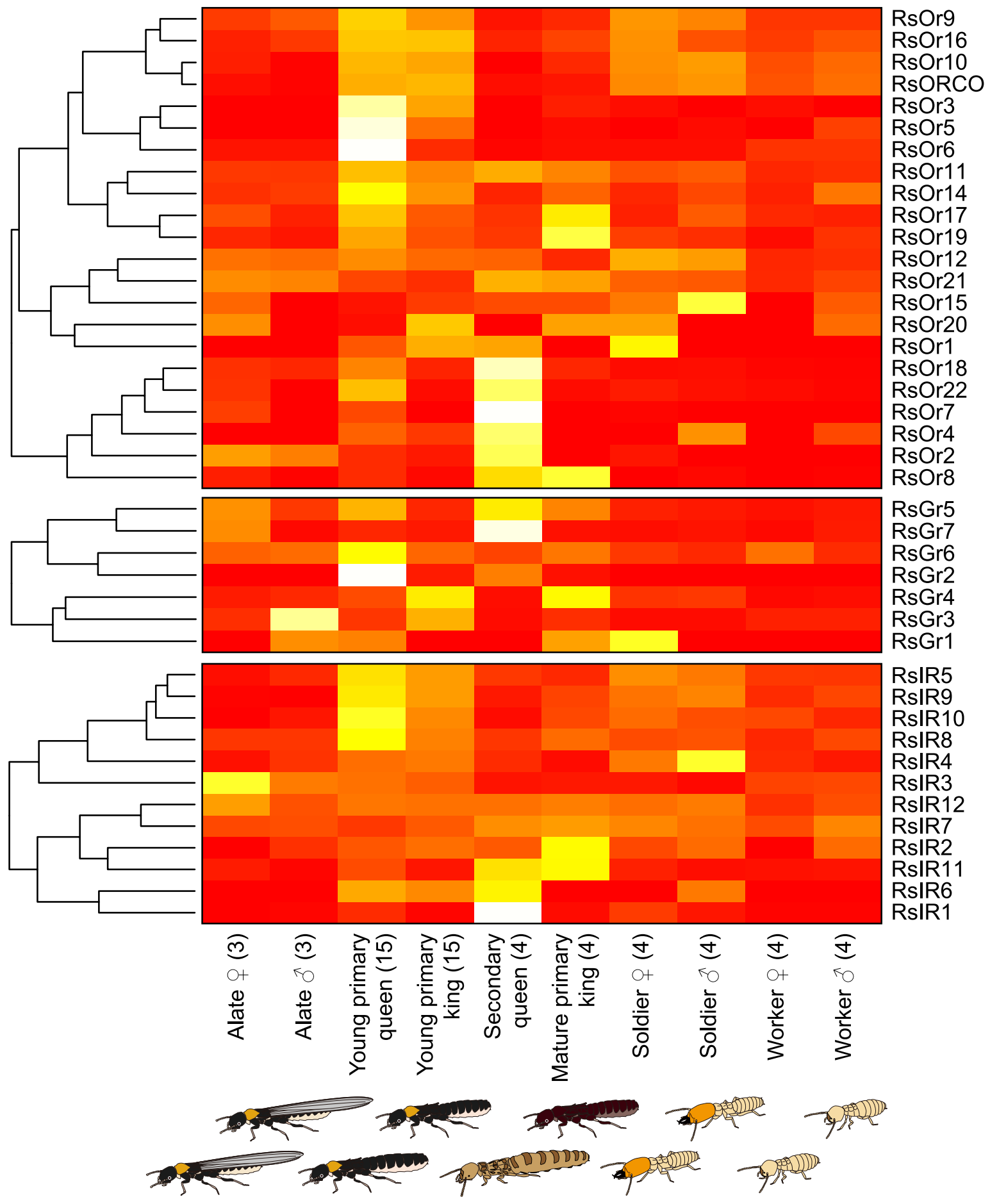

Fig 3. Differential expression of chemoreceptors among castes. The heatmaps exhibit the differential expression of 22 OR, 7 GR, and 12 IR genes among termite castes. Relative expression level indicates the mean normalized Count per Million (CPM), ranging from red (scaled expression of 0 ) to white (scaled expression of 2.5). The tree at the left corresponds to hierarchical clustering of cluster-averaged expression. Numbers in parenthesis after caste names refer to the numbers of biological replication. Ten individuals were pooled for each sex of worker and soldier to obtain sufficient amount of RNA, while single individuals were used for RNA extraction of the other castes.

doi:10.1371/journal.pone.0146125.g003 
RsOr3

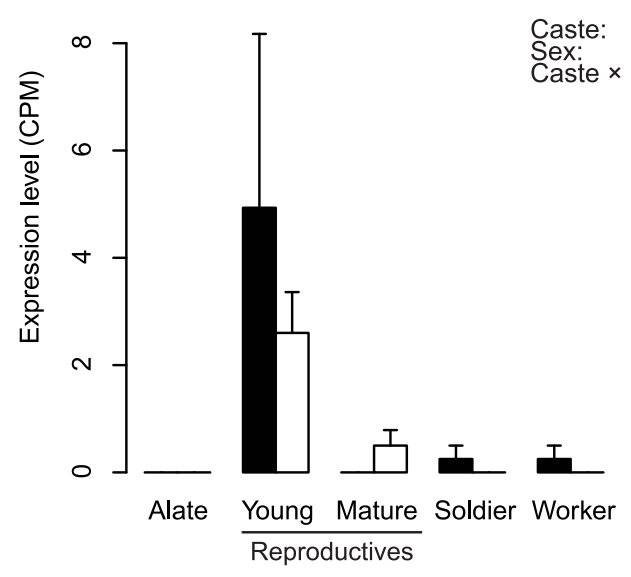

RsGr5

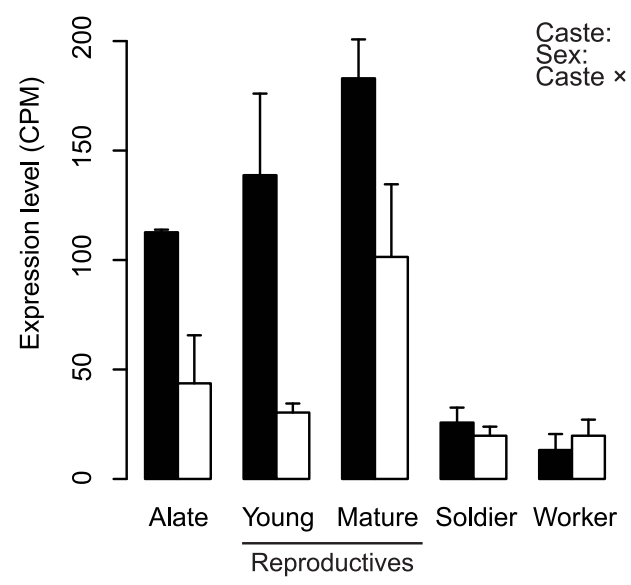

RsIR1

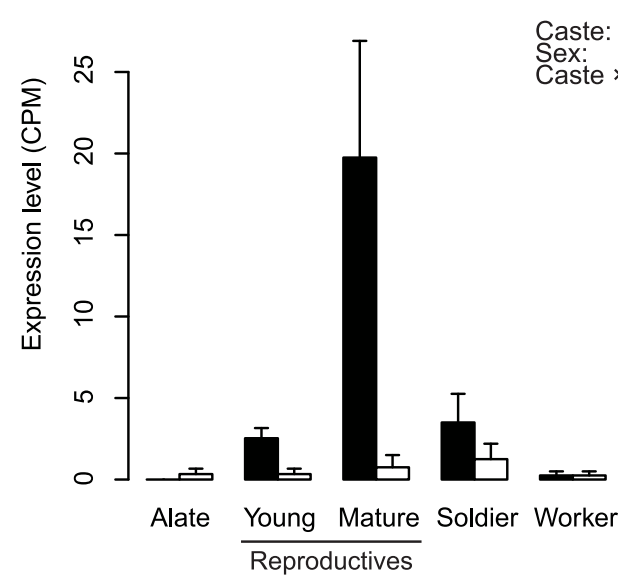

RsGr2

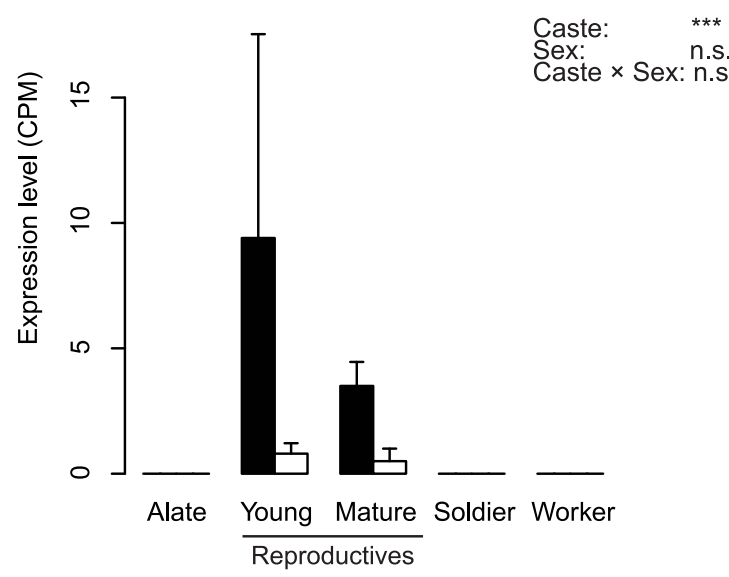

RsGr7

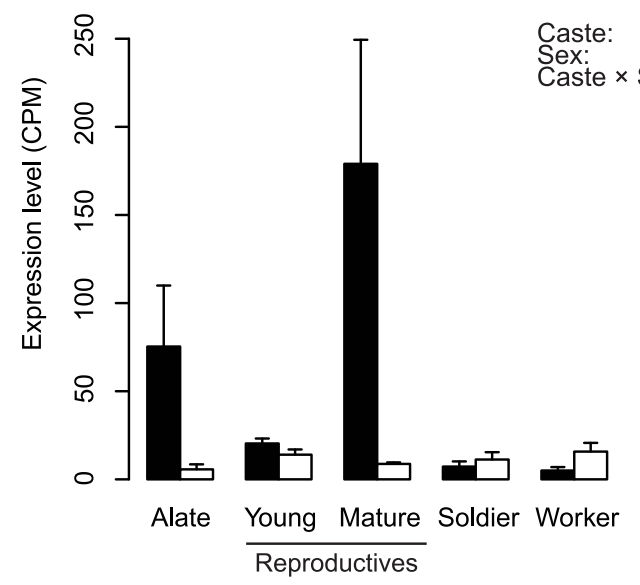

RsIR11

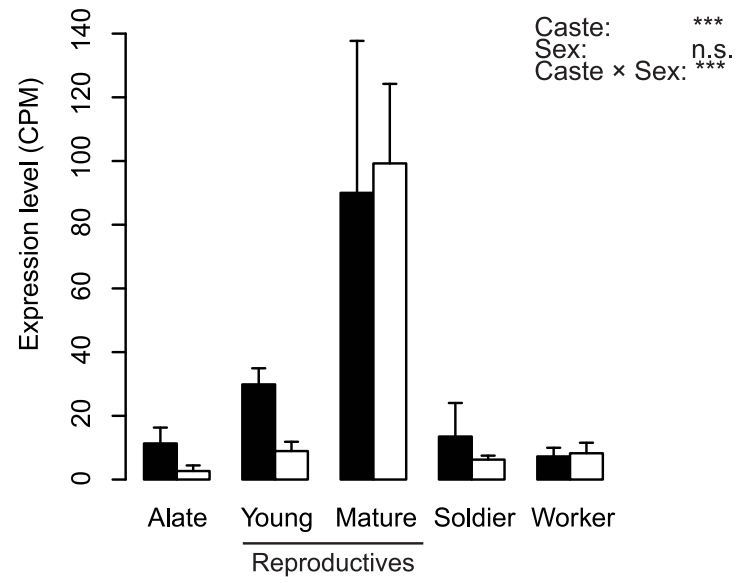

Fig 4. Characteristic expression patterns of ligand-predicted chemoreceptors. Comparison of the mean CPM of six receptor transcripts among castes and between the sexes (black: female, white: male) of each caste. Young reproductives refer to young PQs and PKs. Mature reproductives indicate mature secondary queens and mature PKs. Error bars denote standard errors. Results of statistical analyses for each gene expression are shown in the upper right side of each graph (n.s.: not significant, *: False Discovery Rate $(F D R)<0.05$, **: FDR $<0.01$, ***: FDR $<0.001$ ). 


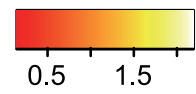

Relative expression level

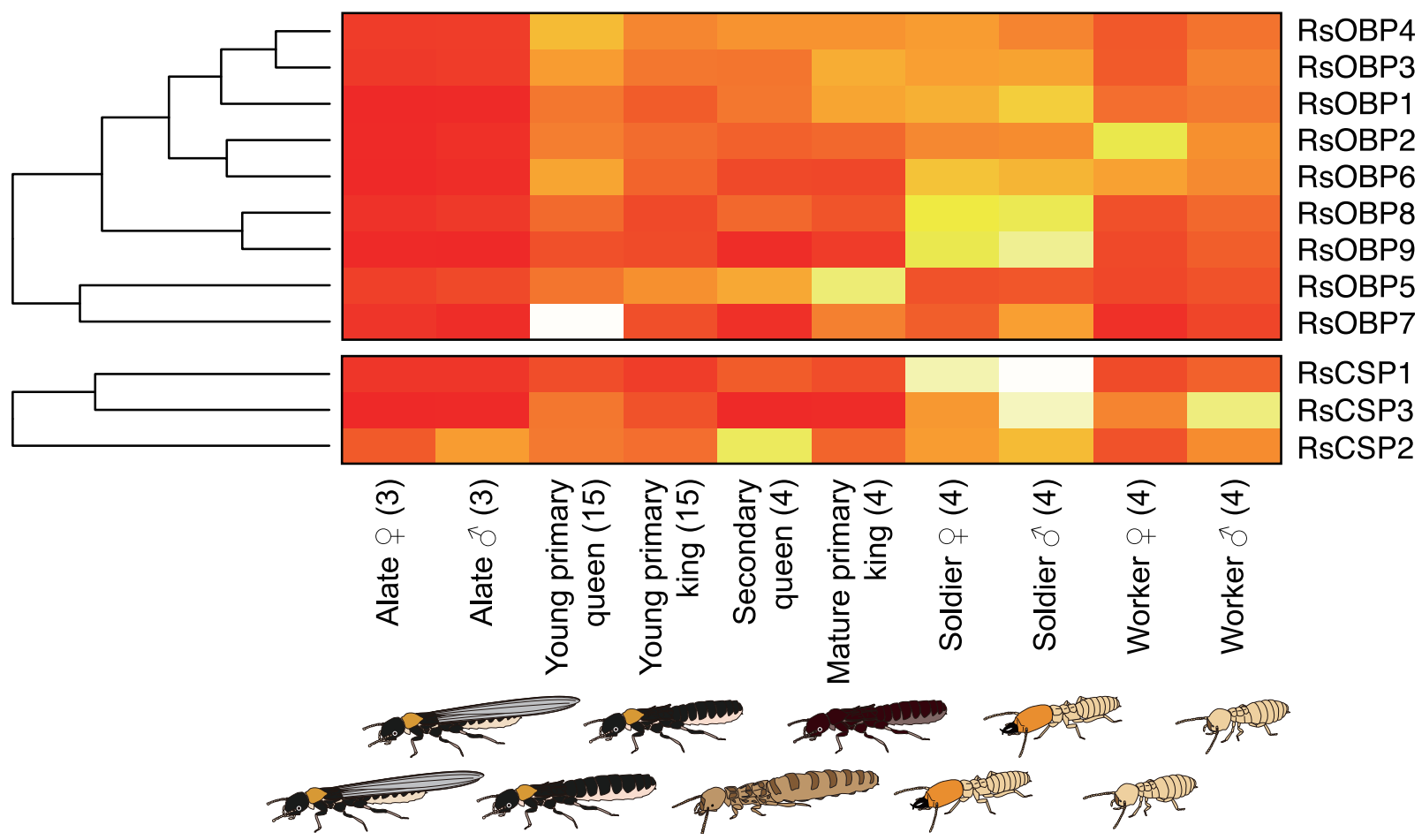

Fig 5. Differential expression of OBP and CSP among castes. The heatmaps exhibit the differential expression of nine OBPs and three CSPs transcripts among termite castes. Abbreviations are referred to in Fig 3. Relative expression level indicates the mean CPM, ranging from red (scaled expression of 0 ) to white (scaled expression of 2). Tree at the left correspond to hierarchical clustering of cluster-averaged expression.

doi:10.1371/journal.pone.0146125.g005

expression level of RsOBP5 was higher in mature primary kings than in other castes (caste: FDR $<0.001$; S6 Fig and S3 Table).

\section{Chemosensory protein}

Three CSPs were predicted, and these showed sequence similarity to those of Holotrichia oblita, Adelphocoris lineolatus, T. castaneum, and B. mori (S2 Table). RsCSP1 was expressed more in soldiers than in other castes (caste: FDR $<0.001$; Fig 5, S6 Fig and S3 Table). RsCSP2 and RsCSP3 showed significant expression among castes (caste: FDR $<0.001$, caste $\times$ sex: FDR $<0.01$; Fig 5, S6 Fig and S3 Table).

\section{Age-related alternation of gene expression levels}

Among the 53 annotated chemoreception-related genes, 26 receptors (13 ORs, 6 GRs and 7 IRs) and 7 binding proteins (7 OBPs and 2 CSPs) showed significant differences among the reproductives of different age classes ( $\mathrm{S} 4 \mathrm{Table}$ ). In male reproductives (male alates, young primary kings, and mature primary kings), young primary kings showed higher expression in 11 receptors (RsOr3, RsOr5, RsOr9, RsOr10, RsORCO, RsOr16, RsIR4, RsIR5, RsIR6, RsIR9, and RsIR10) and 2 binding proteins (RsOBP9 and RsCSP3) than in other age classes (FDR $<0.05$; S2, S5 and S6 Figs and S4 Table), and mature primary kings showed the highest expression in 5 receptors (RsOr8, RsOr17, RsOr19, RsGr5, RsIR11) and 4 OBPs (RsOBP1, RsOBP3, RsOBP5, 
RsOBP7) (FDR < 0.01, Fig 4, S6 Fig and S4 Table). In female reproductives, young primary queens showed higher expression in 9 receptors (RsOr3, RsOr5, RsOr12, RsORCO, RsGr2, RsGr5, RsIR5, RsIR9, RsIR10) and 6 binding proteins (RsOBP1, RsOBP4, RsOBP6, RsOBP7, RsOBP9 and RsCSP3) than in female alates (FDR $<0.05$, S2, S4, S5 and S6 Figs and S4 Table).

\section{Discussion}

Using our RNA-seq dataset, which included RNA assayed from individuals of different castes, sexes, and ages of $R$. speratus, we detected 156,276 ORFs. Based on the subsequent homology search of the known amino acid sequence data of insects (Z. nevadensis, A. pisum, A. mellifera, T. castaneum, B. mori, and D. melanogaster), we inferred 10,238 protein-coding transcripts from the RNA-seq data; among the 10,238 genes, 53 were annotated as chemoreception-related genes consisting of 22 ORs, 7 GRs, 12 IRs 9 OBPs, and 3 CSPs.

Termite societies are characterized by a highly sophisticated caste system with distinct morphological and behavioral differences. Workers perform a variety of tasks including brood and royal care, foraging, nest construction and hygienic activities. In contrast, soldiers and royals are engaged exclusively in nest defense and reproduction, respectively. This indicates that chemosensory demands would be different among castes. As expected, 81\% (43/53) of chemoreception-related genes showed significant caste-specific differences (S3 Table), suggesting adaptation of chemoreception systems for efficient task allocation. The functional significance of the caste-specific expression patterns remains unknown until the ligands of these receptors are identified by future studies.

Among the 53 chemoreception-related genes, 33 genes (62\%) showed significant age-dependent differences in their expression in female and/or male reproductives (S4 Table). In female reproductives, 15 genes showed significantly higher expression levels in young primary queens than in female alates, but 5 receptor genes showed the opposite pattern (FDR $<0.01$, Fig 4, S2 and S5 Figs and S4 Table). In male reproductives, thirteen genes had the highest expression in young primary kings, and nine genes in mature primary king, but four genes were most highly expressed in male alates (FDR $<0.05$, S2, S4, S5 and S6 Figs and S4 Table). These age-dependent expression patterns indicate that many chemoreception-related genes increase expression after the colony is founded.

ORCO interacts with ligand-specific ORs and forms heterodimeric complexes that are essential for trafficking of ORs [26,27]. ORCO is strikingly well conserved across insect species $[28,29]$. In this study, RsORCO showed a high degree of sequence similarity with ORCOs of other insects suggesting an evolutionarily conserved function (S3 Fig and S2 Table). The expression of RsORCO was much higher in individuals engaged in social labor (workers, soldiers and founding pairs) than in those of dispersal stage (alates) and mature reproductive stage (mature king and queens), implying that RsORCO expression may reflect the activity of ORs as a whole. It is important to note that the expression of RsORCO was lower than the total expression of ORs and similar patterns can be seen in the data of the silk moth Bombyx mori [30] and the red flour beetle Tribolium castaneum [31]. Hence, the quantitative relationship between the expression of ORCO and the total expression of ORs remains to be explored in the future studies.

Our analysis of the chemoreceptor genes in the termite has begun to reveal the relationship between division of labor and expression of chemoreceptor genes. In this study we performed the RNA-seq on whole bodies of termites. Therefore, the number of reported chemoreceptor genes might be underestimated, leaving the possibility for future works to find more chemoreceptors showing caste-, sex- or age-specificity. These results provide new insights into the roles 
of chemoreceptors in the division of labor, and provide a foundation for exploring the mechanism and evolution of chemoreceptor polyphenism in termites.

\section{Materials and Methods}

\section{Termites}

To evaluate the expression of chemoreception-related genes, we conducted RNA sequencing (RNA-seq) transcriptome analysis of male and female alates, young primary kings and queens, mature primary kings and mature secondary queens, and male and female soldiers and workers of the subterranean termite R. speratus (Fig 1). To obtain alates, we collected three colonies (colony code: TA130412C, TA130412E, and HI130508N) from secondary forests in Kyoto prefecture, Japan, in the swarming season from April to May 2013 (S1 Table). The alates extracted from the nests were separated by sex, and maintained in Petri dishes containing moist filter paper until they shed their wings. Then, a male and female were randomly selected from each colony and placed in a $90 \mathrm{~mm}$ Petri dish that contained mixed sawdust bait blocks [32]; five nestmate pairs were made for each colony. The Petri dishes were kept at $25^{\circ} \mathrm{C}$ under constant darkness. After 6 months, the nests in bait blocks were dissected to collect young primary queens and kings from the incipient colonies (S1 Table).

To obtain kings and queens from mature colonies of R. speratus, we collected termite nests from secondary forests in Kyoto and Shiga prefecture, Japan, during the reproductive season from July to October 2013. We found royal chambers of four colonies (HI130717B, ZE130827B, $\mathrm{ZE} 130827 \mathrm{H}$, and $\mathrm{YO} 131010 \mathrm{~A}$ ), and carefully extracted kings and queens together with workers and soldiers (S1 Table). All of the kings were primary kings and all of the queens were secondary queens. Workers and soldiers were separated by sex based on the caudal sternite configuration using a stereoscope.

No specific permits were required for the described field studies and no specific permissions were required for the locations/activities for termite sampling because they are public lands and are not privately owned or protected in any way. These field studies did not involve endangered or protected species.

\section{RNA extraction and Illumina sequencing}

Total RNA was extracted from the whole body of each individual of each reproductive caste (alates, kings, and queens) using an RNeasy mini kit (Qiagen), using the standardized instructions from the manufacturer. For workers and soldiers, we pooled 10 individuals of each sex to extract a sufficient amount of RNA for analysis, and performed RNA-seq analysis on a total of 60 samples (DDBJ BioSample ID: SAMD00026264-SAMD00026323). Following previously described procedures [33] for first-strand cDNA synthesis, $9 \mu \mathrm{L}$ mixtures containing more than 50 ng of each total RNA sample and 20 pmol polyT-START primer (AATTGCAGTGG TATCAACGCAGAGCGGCCGCTTTTTTTTTTTTTTTTTTTTTTTTTTTTTVN) were incubated for $3 \mathrm{~min}$ at $65^{\circ} \mathrm{C}$, and then chilled on ice for $3 \mathrm{~min}$. Then, the following were added to each sample: $4.0 \mu \mathrm{L} 5 \mathrm{x}$ first-strand synthesis buffer (Invitrogen), $1.0 \mu \mathrm{L} 10 \mathrm{mM}$ dNTP mix (Promega), $2.0 \mu \mathrm{L}$ 0.1 M DTT (Invitrogen), 2.0 $\mu \mathrm{L} 12 \mu \mathrm{M}$ template-switching RNA primer (AAG CAGUGGUAUCAACGCAGAGUACAUGGG), and $1 \mu \mathrm{L}$ SuperScript II reverse transcriptase (Invitrogen). The samples were incubated for $1 \mathrm{~h}$ at $42^{\circ} \mathrm{C}$, and reactions were terminated by heating at $65^{\circ} \mathrm{C}$ for $15 \mathrm{~min}$. Then, the samples were placed on ice and diluted with $80 \mu \mathrm{L}$ TE buffer prior to cDNA amplification. The cDNA libraries were prepared for second-strand synthesis and amplification. The reaction mixtures were composed of $5 \mu \mathrm{L}$ diluted first strand cDNA, $10 \mu \mathrm{L} 5 \mathrm{x}$ Phusion HF buffer (Finnzymes), $1 \mu \mathrm{L} 10 \mathrm{mM}$ dNTP mix (Promega), $1 \mu \mathrm{L}$ primer pair (10 $\mu$ M each; START: CGCCAGGGTTTTCCCAGTCACGACAATTGCAGTGGTATCAACGCAGA 
and TS_LONG: CTTGTAGGTTAAGTGGAGAGCTAACAATTTCACACAGGAAAGCAGTGG TATCAACGC), and $0.5 \mu \mathrm{L}$ Phusion High-Fidelity DNA polymerase (Finnzymes), and were heated at $98^{\circ} \mathrm{C}$ for $30 \mathrm{~s}$ followed by 15 cycles of $10 \mathrm{~s}$ at $98^{\circ} \mathrm{C}, 6 \mathrm{~min}$ at $68^{\circ} \mathrm{C}$, and finally $10 \mathrm{~min}$ at $72^{\circ} \mathrm{C}$. The PCR products were purified with Dynabeads MyOne Carboxylic acid (Invitrogen) and 16\% PEG6000 with $0.9 \mathrm{M} \mathrm{NaCl}$ and $10 \mathrm{mM}$ Tris- $\mathrm{HCl}$ (pH 6), and $12 \mu \mathrm{L}$ EB buffer was used during the final elution. Tagmentation followed the Nextera XT DNA Sample Prep Kit (illumina) protocol for Hiseq. In January 2014, paired-end 100-cycle multiplex sequencing was performed using the Illumina HiSeq 2000 at the Okinawa Institute of Science and Technology Graduate University. RNA sequence data was deposited in the DNA Data Bank of Japan (DDBJ) under the BioProject "Royal epigenetics in the termite Reticulitermes speratus" (PRJDB3531), which contains links and access to insect sampling data through the BioSample SAMD00026264-SAMD00026323 and the Sequence Read Archive DRR030795DRR030854.

\section{Data processing and de novo transcriptome assembly}

The raw sequencing reads were trimmed by removing adapter sequences. Preprocessing was performed in the cloud-computing based analytical platform, DDBJ Read Annotation Pipeline. Bases with a quality score of less than 20 were trimmed from the 5' and 3' ends of each read. After trimming, reads with a high percentage $(>30 \%)$ of low quality bases $(<15)$ and short reads $(<25 \mathrm{bp}$ ) were discarded, and the remaining reads were used for the assembly. The remaining reads from all of the samples were assembled de novo using Trinity version trinityrnaseq_r2012-04-27 [19, 20], which generates transcriptome assemblies from short read sequences using the de Bruijn graph algorithm. The parameters selected to run Trinity were all default parameters (k-mer length $=25$-mers) except max_reads_per_loop, which was set to 1,500,000. Using transcriptsToOrfs (version 0.0.2), we searched open reading frames (ORFs) in the assembled sequences, and gained predicted amino acid sequences.

\section{Protein-coding prediction}

We converted the predicted amino acid sequences into a searchable database using the formatdb tool of the BLAST+v2.2.29 toolkit (National Center for Biotechnology Information (NCBI), http://www.ncbi.nlm.nih.gov/). To predict gene models using the homology-based method, protein sequences from Z. nevadensis (v2.2, http://termitegenome.org), A. pisum (v2.1b, http://www.aphidbase.com), A. mellifera (v3.2, http://hymenopteragenome.org/ beebase), T. castaneum (v3.0, http://beetlebase.org), B. mori (v2.0, http://silkworm.genomics. org.cn), and D. melanogaster (v6.02, http://flybase.org) were used for BLAST queries with an E-value cutoff of 1e-100.

\section{Annotation of chemoreceptor and binding proteins}

Peptide sequences of chemoreceptors and binding proteins of the following species were obtained from NCBI, and used as BLAST queries for our peptide database with an E-value cutoff of 1E-7: Z. nevadensis [14], P. americana [34], T. castaneum [31, 35, 23], H. oblita [36], D. ponderosae [37], A. mellifera [38, 39], D. melanogaster [17, 40-43], A.gambiae [44], A. aegypti [45], A. lineolatus [46], R. prolixus [47], L. migratria [48], and B. mori [30, 49, 50]. The peptide sequences of ORs of $M$. caryae were translated from the nucleotide sequences [51] using the Nucleotide Sequence Translation tool (EMBL-EBI, http://www.ebi.ac.uk/Tools/st/). 


\section{Abundance estimation and differential expression analyses}

Gene expression levels were estimated by the RSEM version 1.2.8 [52] separately for the filtered reads from each sample. Raw read counts data produced by RSEM were normalized using the Trimmed Mean of M-value (TMM) normalization method [53] and were used for differential expression analyses among castes and between sexes using edgeR package (v3.4.2) [54]. All of the statistical analyses were performed using the R package (v3.0.3) and heatmaps were generated with heatmap. 2 in gplots package.

\section{Supporting Information}

S1 Fig. Length frequency distribution of $R$. speratus contigs. Histogram presentation of sequence-length distribution for significant matches that was found. The horizontal axis indicates sequence sizes from 200 to $\geq 3000$ nucelotides. The vertical axis indicates the number of contigs for each size.

S2 Fig. Expression levels of ORs other than RsOr3. Comparison of the mean CPM of OR transcripts among castes and between the sexes (black: female, white: male) of each caste. Young reproductives refer to young PQs and PKs. Mature reproductives indicate mature secondary queens and mature PKs. Error bars denote standard errors. Results of statistical analyses for each gene expression are shown in the upper right side of each graph (n.s.: not significant, ${ }^{*}$ : FDR $<0.05,{ }^{* *}$ : FDR $<0.01,{ }^{* * *}$ : FDR $\left.<0.001\right)$. (EPS)

S3 Fig. Phylogenetic tree of ORCO sequences from various insects. The tree was constructed based on the ORCO sequence of R. speratus and that of other insect species obtained from the protein database of NCBI. The evolutionary history was inferred using the Maximum Likelihood method based on the JTT matrix-based model [55]. The tree with the highest log likelihood (-12311.7185) is shown. The bootstrap values (1,000 resampling) are shown on the branches. Initial tree(s) for the heuristic search were obtained by applying the Neighbor-Joining method to a matrix of pairwise distances estimated using a JTT model. The tree is drawn to scale, with branch lengths measured in the number of substitutions per site. The analysis involved 84 amino acid sequences. All of the positions containing gaps and missing data were eliminated. There were a total of 382 positions in the final dataset. Evolutionary analyses were conducted in MEGA6 [56]. Branch lengths are proportional. The number written before each scientific name is accession numbers. (EPS)

S4 Fig. Expression levels of GRs other than RsGr2, RsGr5, and RsGr7. Comparison of the mean CPM of GR transcripts among castes and between the sexes (black: female, white: male) of each caste. Young reproductives refer to young PQs and PKs. Mature reproductives indicate mature secondary queens and mature primary kings. Error bars denote standard errors. Results of statistical analyses for each gene expression are shown in the upper right side of each graph (n.s.: not significant, ${ }^{*}$ : FDR $<0.05,{ }^{* *}$ : FDR $<0.01,{ }^{* * *}$ : FDR $<0.001$ ). (EPS)

S5 Fig. Expression levels of IRs other than RsIR1 and RsIR11. Comparison of the mean CPM of IR transcripts among castes and between the sexes (black: female, white: male) of each caste. Young reproductives are referred to as young PQs and PKs. Mature reproductives indicate mature secondary queens and mature PKs. Error bars denote standard errors. Results of statistical analyses for each gene expression are shown in the upper right side of each graph 
(n.s.: not significant, ${ }^{*}$ : FDR $<0.05,{ }^{* *}$ : FDR $<0.01,{ }^{* * *}$ : FDR $<0.001$ ). (EPS)

S6 Fig. Expression levels of OBPs and CSPs. Comparison of the mean CPM of OBP and CSP transcripts among castes and between the sexes (black: female, white: male) of each caste. Young reproductives are referred to as young PQs and PKs. Mature reproductives indicate mature secondary queens and mature PKs. Error bars denote standard errors. Results of statistical analyses for each gene expression are shown in the upper right side of each graph (n.s.: not significant, ${ }^{*}$ : FDR $<0.05,{ }^{* *}$ : FDR $<0.01,{ }^{* * *}$ : FDR $\left.<0.001\right)$. (EPS)

S1 Table. RNA-seq sample statistics. Numbers in colony codes indicate the dates when the colonies were collected (e.g. colony TA130412C was collected on April 12, 2013). *: Ten individuals were pooled for each sex of worker and soldier to obtain sufficient amount of RNA, while single individuals were used for RNA extraction of the other castes. (DOC)

S2 Table. Summary results of blast homology searches. Blast homology searches of amino acid sequences in $R$. speratus were performed on the sequences of a grasshopper (L. migratoria), a cockroach (P. americana), a termite ( $Z$. nevadensis), bugs (R. prolixus and $A$. lineolatus), a honeybee (A. mellifera), beetles (T. castaneum, M. caryae, H. oblita, and D. ponderosae), a silkworm (B. mori), mosquitoes (A.gambiae and A. aegypti), and a fruit fly (D. melanogaster).

(DOCX)

S3 Table. Statistical results of differential expression levels among castes and sexes for each gene. Comparison of normalized counts per million (CPM) among castes and sexes was conducted using edgeR package. Bold letters means significant difference (FDR $<0.05)$. LR: likelihood ratio, FDR: false discovery rate, OR: odorant receptor, GR: gustatory receptor, IR: ionotropic receptor, OBP: odorant-binding protein, CSP: chemosensory protein.

(DOCX)

S4 Table. Statistical results of age-related expression changes in male or female reproductives. Comparison of normalized counts per million (CPM) among male reproductives (alates, young and mature primary kings) or female ones (alate and young primary queens) was conducted using edgeR package. Bold letters means significant difference (FDR $<0.05$ ). Abbreviations were referred to S3 Table.

(DOCX)

\section{Acknowledgments}

We thank C. Iwata, N. Mizumoto, Y. Namba, T. Nozaki, and W. Suehiro for research assistance; T. Fuchikawa for technical advice; National Institute of Genetics (NIG) for utilizing super computer system.

\section{Author Contributions}

Conceived and designed the experiments: KK KM YM. Performed the experiments: KK MMYT AM YW. Analyzed the data: YM KK. Contributed reagents/materials/analysis tools: KK KM. Wrote the paper: YM KK KM. 


\section{References}

1. Wyatt TD. Pheromones and animal behavior: Communication by smell and taste. 1st ed. Cambridge: Cambridge University Press; 2003.

2. Karlson $P$, Lüscher M. Pheromones-New term for a class of biologically active substances. Nature. 1959; 183: 55-56. PMID: 13622694

3. Wilson EO. Chemical communication in the social insects-Insect societies are organized principally by complex systems of chemical signals. Science. 1965; 149: 1064-1071.

4. Wilson EO. The insect societies. 1st ed. Cambridge: Harvard University Press; 1971.

5. Keller L, Nonacs $P$. The role of queen phoromones in social insects: queen control or queen signal? Anim Behav. 1993; 45: 787-794.

6. Slessor KN, Winston ML, Le Conte Y. Pheromone communication in the honeybee (Apis mellifera L.). $J$ Chem Ecol. 2005; 31: 2731-2745. PMID: 16273438

7. Light SF. Experimental studies on ectohormonal control of the development of supplementary reproductives in the termite genus Zootermopsis (formerly Termopsis). 1st ed. Oakland: University of California Press; 1944.

8. Lücher M. Die produktion und elimination von ersatzgeschlechtstieren bei der termite Kalotermes flavicollis Fabr. Z Vergl Physiol. 1952; 34: 123-141.

9. Lücher M. Social control of polymorphism in termites. 1st ed. London: Royal entomological society; 1961.

10. Matsuura K, Himuro C, Yokoi T, Yamamoto Y, Vargo EL, Keller L. Identification of a pheromone regulating caste differentiation in termites. P Natl Acad Sci U S A. 2010; 107: 12963-12968.

11. Matsuura K, Vargo EL, Kawatsu K, Labadie PE, Nakano H, Yashiro T, et al. Queen succession through asexual reproduction in termites. Science. 2009; 323: 1687-1687. doi: 10.1126/science.1169702 PMID: 19325106

12. Yashiro $T$, Matsuura $K$. Termite queens close the sperm gates of eggs to switch from sexual to asexual reproduction. P Natl Acad Sci U S A. 2014; 111: 17212-17217.

13. Yamamoto $Y$, Matsuura $K$. Genetic influence on caste determination underlying the asexual queen succession system in a termite. Behav Ecol Sociobiol. 2012; 66: 39-46.

14. Terrapon N, Li C, Robertson HM, Ji L, Meng X, Booth W, et al. Molecular traces of alternative social organization in a termite genome. Nat Commun. 2014; 5: 3636. doi: 10.1038/ncomms4636 | www. nature.com/naturecommunications. PMID: 24845553

15. Jacquin-Joly E, Merlin C. Insect olfactory receptors: Contributions of molecular biology to chemical ecology. J Chem Ecol. 2004; 30: 2359-2397. PMID: 15724962

16. Vosshall LB, Stocker RE. Molecular architecture of smell and taste in Drosophila. Annu Rev Neurosci. 2007; 30: 505-533. PMID: 17506643

17. Rytz R, Croset V, Benton R. Ionotropic Receptors (IRs): Chemosensory ionotropic glutamate receptors in Drosophila and beyond. Insect Biochem Molec. 2013; 43: 888-897.

18. Zhang H-J, Anderson AR, Trowell SC, Luo A-R, Xiang Z-H, Xia Q-Y. Topological and functional characterization of an insect gustatory receptor. Plos One. 2011; 6: e24111. doi: 10.1371/journal.pone. 0024111 PMID: 21912618

19. Haas BJ, Papanicolaou A, Yassour M, Grabherr M, Blood PD, Bowden J, et al. De novo transcript sequence reconstruction from RNA-seq using the Trinity platform for reference generation and analysis. Nat Protoc. 2013; 8: 1494-1512. doi: 10.1038/nprot.2013.084 PMID: 23845962

20. Grabherr MG, Haas BJ, Yassour M, Levin JZ, Thompson DA, Amit I, et al. Full-length transcriptome assembly from RNA-Seq data without a reference genome. Nat Biotechnol. 2011; 29: 644-654. doi: 10.1038/nbt.1883 PMID: 21572440

21. Richards S, Gibbs RA, Gerardo NM, Moran N, Nakabachi A, Stern D, et al. Genome sequence of the pea aphid Acyrthosiphon pisum. Plos Biology. 2010; 8: e1000313. doi: 10.1371/journal.pbio.1000313 PMID: 20186266

22. Weinstock GM, Robinson GE, Gibbs RA, Worley KC, Evans JD, Maleszka R, et al. Insights into social insects from the genome of the honeybee Apis mellifera. Nature. 2006; 443: 931-949. PMID: 17073008

23. Richards S, Gibbs RA, Weinstock GM, Brown SJ, Denell R, Beeman RW, et al. The genome of the model beetle and pest Tribolium castaneum. Nature. 2008; 452: 949-955. doi: 10.1038/nature06784 PMID: 18362917

24. Xia Q, Wang J, Zhou Z, Li R, Fan W, Cheng D, et al. The genome of a lepidopteran model insect, the silkworm Bombyx mori. Insect Biochem Molec. 2008; 38:1036-1045. 
25. Adams MD, Celniker SE, Holt RA, Evans CA, Gocayne JD, Amanatides PG, et al. The genome sequence of Drosophila melanogaster. Science. 2000; 287: 2185-2195. PMID: 10731132

26. Larsson MC, Domingos AI, Jones WD, Chiappe ME, Amrein H, Vosshall LB. Or83b encodes a broadly expressed odorant receptor essential for Drosophila olfaction. Neuron. 2004; 43: 703-714. PMID: 15339651

27. Benton R, Sachse S, Michnick SW, Vosshall LB. Atypical membrane topology and heteromeric function of Drosophila odorant receptors in vivo. Plos Biology. 2006; 4: 240-257.

28. Hallem EA, Dahanukar A, Carlson JR. Insect odor and taste receptors. Annu. Rev. Entomol. 2006; 51: 113-135. PMID: 16332206

29. Sato K, Touhara K. Insect seven-transmembrane olfactory receptor complex is an odor-gated ion channel. Nihon yakurigaku zasshi. Folia pharmacologica Japonica. 2009; 134: 248-253.

30. Wanner KW, Anderson AR, Trowell SC, Theilmann DA, Robertson HM, Newcomb RD. Female-biased expression of odourant receptor genes in the adult antennae of the silkworm, Bombyx mori. Insect Mol Biol. 2007; 16: 107-119. PMID: 17257213

31. Abdel-Latief M. A family of chemoreceptors in Tribolium castaneum (Tenebrionidae: Coleoptera). Plos One. 2007; 2: e1319. PMID: 18091992

32. Matsuura K, Nishida T. Colony fusion in a termite: What makes the society "open"? Insect Soc. 2001; 48: 378-383.

33. Matz M, Shagin D, Bogdanova E, Britanova O, Lukyanov S, Diatchenko L, et al. Amplification of cDNA ends based on template-switching effect and step-out PCR. Nucleic Acids Res. 1999; 27: 1558-1560. PMID: 10037822

34. Xu Y-L, He P, Zhang L, Fang S-Q, Dong S-L, Zhang Y-J, et al. Large-scale identification of odorantbinding proteins and chemosensory proteins from expressed sequence tags in insects. BMC Genomics. 2009; 10: 632. doi: 10.1186/1471-2164-10-632 PMID: 20034407

35. Engsontia P, Sandersona AP, Cobb M, Walden KKO, Robertson HM, Brown S. The red flour beetle's large nose: An expanded odorant receptor gene family in Tribolium castaneum. Insect Biochem Molec. 2008; 38: 387-397.

36. Sun $\mathrm{H}$, Guan L, Feng H, Yin J, Cao Y, Xi J, et al. Functional characterization of chemosensory proteins in the scarab beetle, Holotrichia oblita Faldermann (Coleoptera: Scarabaeida). Plos One. 2014; 9: e107059. doi: 10.1371/journal.pone.0107059 PMID: 25188038

37. Andersson MN, Grosse-Wilde E, Keeling Cl, Bengtsson JM, Yuen MMS, Li M, et al. Antennal transcriptome analysis of the chemosensory gene families in the tree killing bark beetles, Ips typographus and Dendroctonus ponderosae (Coleoptera: Curculionidae: Scolytinae). BMC Genomics. 2013; 14: 198. doi: 10.1186/1471-2164-14-198 PMID: 23517120

38. Robertson HM, Wanner KW. The chemoreceptor superfamily in the honey bee, Apis mellifera: Expansion of the odorant, but not gustatory, receptor family. Genome Res. 2006; 16: 1395-1403. PMID: 17065611

39. Forêt S, Wanner KW, Maleszka R. Chemosensory proteins in the honey bee: Insights from the annotated genome, comparative analyses and expressional profiling. Insect Biochem Molec. 2007; 37: 1928.

40. Abuin L, Bargeton B, Ulbrich MH, Isacoff EY, Kellenberger S, Benton R. Functional architecture of olfactory ionotropic glutamate receptors. Neuron. 2011; 69: 44-60. doi: 10.1016/j.neuron.2010.11.042 PMID: 21220098

41. Benton R, Vannice KS, Gomez-Diaz C, Vosshall LB. Variant ionotropic glutamate receptors as chemosensory receptors in Drosophila. Cell. 2009; 136: 149-162. doi: 10.1016/j.cell.2008.12.001 PMID: 19135896

42. Clyne PJ, Warr CG, Freeman MR, Lessing D, Kim JH, Carlson JR. A novel family of divergent seventransmembrane proteins: Candidate odorant receptors in Drosophila. Neuron. 1999; 22: 327-338. PMID: 10069338

43. Pikielny CW, Hasan G, Rouyer F, Rosbash M. Members of a family of Drosophila putative odorant-binding proteins are expressed in different subsets of olfactory hairs. Neuron. 1994; 12: 35-49. PMID: 7545907

44. Holt RA, Chaturvedi K. The genome sequence of the malaria mosquito Anopheles gambiae. Science. 2002; 298: 129-149. PMID: 12364791

45. McBride CS, Baier F, Omondi AB, Spitzer SA, Lutomiah J, Sang R, et al. Evolution of mosquito preference for humans linked to an odorant receptor. Nature. 2014; 515: 222-231. doi: 10.1038/nature13964 PMID: 25391959 
46. Gu S-H, Wang S-Y, Zhang X-Y, Ji P, Liu J-T, Wang G-R, et al. Functional characterizations of chemosensory proteins of the alfalfa plant bug Adelphocoris lineolatus indicate their involvement in host recognition. Plos One. 2012; 7: e42871. doi: 10.1371/journal.pone.0042871 PMID: 22900060

47. Ribeiro JMC, Genta FA, Sorgine MHF, Logullo R, Mesquita RD, Paiva-Silva GO, et al. An insight into the transcriptome of the digestive tract of the bloodsucking bug, Rhodnius prolixus. PLoS Negl Trop Dis. 2014; 8: e2594. doi: 10.1371/journal.pntd.0002594 PMID: 24416461

48. Jin X, Zhang SG, Zhang L. Expression of odorant-binding and chemosensory proteins and spatial map of chemosensilla on labial palps of Locusta migratoria (Orthoptera: Acrididae). Arthropod Struct Dev. 2006; 35: 47-56. PMID: 18089057

49. Sakurai T, Nakagawa $\mathrm{T}$, Mitsuno $\mathrm{H}$, Mori H, Endo $\mathrm{Y}$, Tanoue $\mathrm{S}$, et al. Identification and functional characterization of a sex pheromone receptor in the silkmoth Bombyx mori. P Natl Acad Sci U S A. 2004; 101: 16653-16658.

50. Gong D-P, Zhang H-J, Zhao $\mathrm{P}$, Xia Q-Y, Xiang Z-H. The odorant binding protein gene family from the genome of silkworm, Bombyx mori. BMC Genomics. 2009; 10: 332. doi: 10.1186/1471-2164-10-332 PMID: 19624863

51. Mitchell RF, Hughes DT, Luetje CW, Millar JG, Soriano-Agatón F, Hanks LM, et al. Sequencing and characterizing odorant receptors of the cerambycid beetle Megacyllene caryae. Insect Biochem Molec. 2012; 42: 499-505.

52. Li B, Dewey CN. RSEM: accurate transcript quantification from RNA-Seq data with or without a reference genome. BMC Bioinformatics. 2011; 12: 323. doi: 10.1186/1471-2105-12-323 PMID: 21816040

53. Robinson MD, Oshlack A. A scaling normalization method for differential expression analysis of RNAseq data. Genome Biology. 2010; 11:R25. doi: 10.1186/gb-2010-11-3-r25 PMID: 20196867

54. Robinson MD, McCarthy DJ, Smyth GK. edgeR: a Bioconductor package for differential expression analysis of digital gene expression data. Bioinformatics. 2010; 26: 139-140. doi: 10.1093/ bioinformatics/btp616 PMID: 19910308

55. Jones DT, Taylor WR, Thornton JM. The rapid generation of mutation data matrices from protein sequences. Comput Appl Biosci. 1992; 8: 275-282. PMID: 1633570

56. Tamura K, Stecher G, Peterson D, Filipski A, Kumar S. MEGA6: Molecular Evolutionary Genetics Analysis Version 6.0. Mol Biol Evol. 2013; 30: 2725-2729. doi: 10.1093/molbev/mst197 PMID: 24132122 